\title{
A uniqueness theorem for eigenvalue problem having special potential type
}

\author{
Erdal Bas*, Etibar S. Panakhov, Resat Yilmazer \\ Department of Mathematics, Faculty of Science, Firat University, Elazig, 23119, Turkey.
}

Communicated by H. M. Baskonus

\begin{abstract}
In this study, a uniqueness theorem is given for Sturm-Liouville problem with special singular potential. We prove that singular potential function can be uniquely determined by the spectral set $\left\{\lambda_{n}\left(q_{0}, h_{m}\right)\right\}_{m=1}^{+\infty}$. (C)2017 All rights reserved.
\end{abstract}

Keywords: Singular, Sturm Liouville, uniqueness theorem, eigenvalue.

2010 MSC: 34BO5, 47E05.

\section{Introduction}

Inverse problems are the problems that consist of finding an unknown property of an object or medium from the observation of a response of this object or medium, to a probing signal. Inverse problems, in particularly self-adjoint ones, received extensive in last times such problems often emerge in mathematics, mechanics, physics, electronics, geophysics and many branches of science world.

Let $\ell=-\frac{\mathrm{d}^{2}}{\mathrm{~d} \mathrm{x}^{2}}+\mathrm{q}(\mathrm{x})$ be the Sturm-Liouville operator defined by the Dirichlet boundary conditions as self-adjoint operator in $\mathrm{L}^{2}[0,1]$ and $0<\lambda_{1}<\lambda_{2}<\cdots$ be its eigenvalues. Spectral datas are spectra, eigenfunction, normalizing constants, spectral functions, scattering data, etc. An early important result in this direction which gave vital impetus for the further development of inverse problem theory, was obtained. Inverse problems from two spectra were the most simple in their formulation and well-studied in $[1,3]$. Marčenko and Levitan used the transformation to prove that the eigenvalues and norming constants uniquely determine potential function $[13,15]$. Some effective methods of constructing a regular and singular Sturm-Liouville operator for a spectral function or for two spectra are given [2, 8, 11, 19]. We note that the detail of inverse problem for singular equations are given in the monographs and references therein $[5,7,9,17,18]$.

In some recent interesting works, $[10,12,14]$ have taken a different approach to inverse spectral theory for the Sturm-Liouville problem. And then inverse problems have been studied by several authors [20, 21].

As a result, these types of studies created a big impact on the world of natural science. The works still continue intensive on inverse spectral problem. We mentioned that analogous results are given by Mclaughlin and Rundell in [16].

In our study, we will deal with a uniqueness theorem for Sturm-Liouville operator having special singularity type. We show that if the spectral set $\left\{\left[\lambda_{n}\left(q_{0}, h_{m}\right)\right]_{m=1}^{+\infty}, n=0,1,2, \cdots\right\}$ for distinct $h_{m}$ can

\footnotetext{
*Corresponding author

Email address: erdalmat@yahoo.com (Erdal Bas)
} 
be measured, then the spectral set is sufficient to determine the potential $q(x)=\frac{\delta}{x^{p}}+q_{0}(x)$.

In this section, we will focus on some important facts which will be used in this research.

\section{Preliminary knowledge}

Lemma 2.1 (Riemann-Lebesgue's Lemma [4]). If $\mathrm{f}$ is Lebesque integrable on $[-\pi, \pi]$, then

$$
\lim _{n \rightarrow \infty} \int_{-\pi}^{\pi} f(x) \cos n x d x=0=\lim _{n \rightarrow \infty} \int_{-\pi}^{\pi} f(x) \sin n x d x .
$$

We consider the singular Sturm-Liouville problem as follows

$$
\begin{gathered}
-y^{\prime \prime}+\left[\frac{\delta}{x^{p}}+q_{0}(x)\right] y=\lambda y,\left(\mu^{2}=\lambda, 0<x \leqslant \pi\right), \\
y(0)=0 \\
y^{\prime}(\pi)-h y(\pi)=0 \\
y^{\prime}(\pi)-h_{m} y(\pi)=0
\end{gathered}
$$

where $q(x)=\frac{\delta}{x^{p}}+q_{0}(x), \int_{0}^{\pi} x|q(x)| d x<\infty, \delta=$ constant, $q_{0}(x) \in L_{2}(0, \pi], 1<p<2, h, h_{m} \in \mathbb{R}$, $h_{m}<h_{m+1},(m=1,2, \cdots)$.

Similarly, we consider second problem as

$$
\begin{gathered}
-\tilde{y}^{\prime \prime}+\left[\frac{\delta}{x^{p}}+\tilde{q}_{0}(x)\right] \tilde{y}=\mu \tilde{y}, \quad\left(\mu^{2}=\lambda, 0<x \leqslant \pi\right), \\
\tilde{y}(0)=0, \\
\tilde{y}^{\prime}(\pi)-h_{m} \tilde{y}(\pi)=0,
\end{gathered}
$$

where the problem has above similar properties. Let $\lambda_{n}^{\mathrm{D}}(q)$ be the $n$-th eigenvalue for the equation (2.1) with Dirichlet boundary conditions.

The authors in [12, 14, 20,21] showed the recostruction of the potential function for regüler Sturm Liouville problem according to normalizing constants and eigenvalues. Our aim is to apply the same method for singular Sturm Liouville problem.

In this section, we will try to obtain some asymptotic results and a reconstruction formula for potential $\mathrm{q}$, which has been obtained as solution of an inverse nodal problem.

Lemma $2.2([5,7,17])$.

$$
\lambda_{n}=n-\frac{1}{2}+\frac{k}{\left(n-\frac{1}{2}\right)}+O\left(\frac{1}{n^{4-2 p}}\right),
$$

where $\mathrm{k}=\frac{\frac{1}{2} \int_{0}^{\pi}\left(\frac{\delta}{\mathrm{t}^{\mathrm{p}}}+\mathrm{q}_{0}(\mathrm{t})\right) \mathrm{dt}-\mathrm{h}}{\pi}$.

Lemma 2.3. Let $\lambda_{n}$ be the $n$-th eigenvalue of the boundary problem (2.1)-(2.3), for a fixed index $n$, then $\lambda_{n}$ satisfies

$$
\lambda_{\mathrm{n}}^{\mathrm{D}}<\lambda_{\mathrm{n}}<\lambda_{\mathrm{n}+1}^{\mathrm{D}} \text {. }
$$

Theorem 2.4 ([17]). Solution of the boundary value problem (2.5)-(2.6) is

$$
y(x, \lambda) \equiv \frac{\sin \lambda x}{\lambda}+\int_{0}^{x} K(x, s) \frac{\sin \lambda s}{\lambda} d s,
$$

where the kernels $\mathrm{K}(\mathrm{x}, \mathrm{s})$ is the solution of the equation 


$$
\frac{\partial^{2} K}{\partial x^{2}}-\left[\frac{\delta}{x^{p}}+\tilde{q}_{0}(x)\right] K=\frac{\partial^{2} K}{\partial s^{2}}-\left[\frac{\delta}{s^{p}}+q_{0}(s)\right] K,
$$

with conditions

$$
\mathrm{K}(x, x)=\frac{1}{2} \int_{0}^{x}\left(\tilde{\mathrm{q}}_{0}(\mathrm{~s})-\mathrm{q}_{0}(\mathrm{~s})\right) \mathrm{ds}, \quad \mathrm{K}(x, 0)=0 .
$$

Now, merely one of the kinds of the inverse problem is solved. We give the solution of the inverse problem for special singularity potential as follows.

Theorem 2.5 ([6]). The equation (2.1) has fundamental $\varphi(x, \lambda)$ and $\Psi(x, \lambda)$ solutions that satisfies the following asymptotic formulas

$$
\begin{gathered}
\varphi(x, \lambda)=x[1+o(1)], \quad \varphi^{\prime}(x, \lambda)=1+o(1), \\
\psi(x, \lambda)=1+o(1), \quad \psi^{\prime}(x, \lambda)=o\left(\frac{1}{x}\right),
\end{gathered}
$$

for each eigenvalue $\lambda$ and $x \rightarrow 0$ then the entire function $\varphi(x, \lambda)$ with respect to $\lambda$ and $x \geqslant 0$ provides the following inequalities

$$
\begin{gathered}
|\varphi(x, \lambda)| \leqslant x e^{|\operatorname{Im} \lambda| x} \exp \left\{\int_{0}^{x} s|q(s)| d s\right\}, \\
\left|\varphi(x, \lambda)-\frac{\sin \lambda x}{\lambda}\right| \leqslant x \int_{0}^{x} s|q(s)| d s \exp \left\{|\operatorname{Im} \lambda| x+\int_{0}^{x} s|q(s)| d s\right\}, \\
|\lambda \varphi(x, \lambda)-\sin \lambda x| \leqslant\left[\sigma_{1}(0)-\sigma_{1}\left(\frac{1}{\lambda}\right)\right] \exp \left\{|\operatorname{Im} \lambda| x+\int_{0}^{x} s|q(s)| d s\right\},
\end{gathered}
$$

where

$$
\sigma_{1}(x)=\int_{x}^{\pi} \sigma(s) d s, \quad \sigma(x)=\int_{x}^{\pi}|q(s)| d s .
$$

Theorem 2.6. Let $\lambda_{n}\left(q_{0}, h\right)$ be $n$-th eigenvalue of the boundary-value problem (2.1)-(2.3). For a fixed index $n$, then eigenvalue $\lambda_{\mathrm{n}}\left(\mathrm{q}_{0}, \mathrm{~h}\right)$ is strictly increasing in $\mathrm{h}$ and

$$
\lambda_{n}^{D}\left(q_{0}\right)<\lambda_{n}\left(q_{0}, h_{1}\right)<\lambda_{n}\left(q_{0}, h_{2}\right)<\cdots<\lambda_{n}\left(q_{0}, h_{m}\right)<\cdots<\lambda_{n+1}^{D}\left(q_{0}\right) .
$$

Proof. We see that $\lambda_{n}\left(q_{0}, h\right)$ is a continuous function in $h$. Now we prove that $\lambda_{n}\left(q_{0}, h\right)$ is monotonous function in $h$. Using Lemma 2.3, we have

$$
\begin{gathered}
-y^{\prime \prime}\left(x, \lambda_{n}\left(q_{0}, h\right)\right)+\left[\frac{\delta}{x^{p}}+q_{0}(x)\right] y\left(x, \lambda_{n}\left(q_{0}, h\right)\right)=\lambda\left(q_{0}, h\right) y\left(x, \lambda_{n}\left(q_{0}, h\right)\right), \\
-y^{\prime \prime}\left(x, \lambda_{n}\left(q_{0}, h+\triangle h\right)\right)+\left[\frac{\delta}{x^{p}}+q_{0}(x)\right] y\left(x, \lambda_{n}\left(q_{0}, h+\triangle h\right)\right) \\
=\lambda\left(q_{0}, h+\triangle h\right) y\left(x, \lambda_{n}\left(q_{0}, h+\triangle h\right)\right) .
\end{gathered}
$$

Multiplying (2.9) by y $\left(x, \lambda_{n}\left(q_{0}, h+\Delta h\right)\right),(2.10)$ by $y\left(x, \lambda_{n}\left(q_{0}, h\right)\right)$ and subtracting and integrating from 0 to $\pi$, we obtain

$$
\Delta \lambda_{n}\left(q_{0}, h\right) \int_{0}^{\pi} y\left(x, \lambda_{n}\left(q_{0}, h\right)\right) y\left(x, \lambda_{n}\left(q_{0}, h+\Delta h\right)\right) d x=\Delta h y\left(\pi, \lambda_{n}\left(q_{0}, h\right)\right) y\left(\pi, \lambda_{n}\left(q_{0}, h+\Delta h\right)\right),
$$


where $\Delta \lambda_{n}\left(q_{0}, h\right)=\lambda_{n}\left(q_{0}, h+\Delta h\right)-\lambda_{n}\left(q_{0}, h\right)$.

By dividing (2.11) by $\Delta \mathrm{h}$ and letting $\Delta \mathrm{h} \longrightarrow 0$ in (2.8) we have

$$
\frac{\partial \lambda_{n}\left(q_{0}, h\right)}{\partial h} \int_{0}^{\pi} y^{2}\left(x, \lambda_{n}\left(q_{0}, h\right)\right) d x=y^{2}\left(\pi, \lambda_{n}\left(q_{0}, h\right)\right) .
$$

If $y\left(\pi, \lambda_{n}\left(q_{0}, h\right)\right)=0$, then $y^{\prime}\left(\pi, \lambda_{n}\left(q_{0}, h\right)\right)=0$. According to uniqueness theorem of solution, this yields

$$
y\left(x, \lambda_{n}\left(q_{0}, h\right)\right) \equiv 0
$$

It is contradiction with the eigenfunction $y\left(x, \lambda_{n}\left(q_{0}, h\right)\right) \neq 0$ corresponding to eigenvalue $\lambda_{n}\left(q_{0}, h\right)$. Therefore, $y^{2}\left(x, \lambda_{n}\left(q_{0}, h\right)\right)>0$ and $\int_{0}^{\pi} y^{2}\left(x, \lambda_{n}\left(q_{0}, h\right)\right) d x>0$. By virtue of estimate (2.12) we find that

$$
\frac{\partial \lambda_{n}\left(q_{0}, h\right)}{\partial h}>0
$$

Hence $\lambda_{n}\left(q_{0}, h\right)$ is precisely increasing in $h$. Furthermore,

$$
\lambda_{n}\left(q_{0}, h_{m}\right)<\lambda_{n}\left(q_{0}, h_{m+1}\right), \quad h_{m}<h_{m+1} .
$$

\section{The main theorem}

Theorem 3.1. Let $\lambda_{n}\left(q_{0}, h_{m}\right)$ be the $n$-th eigenvalue of the boundary-value problem (2.1), (2.2), (2.3), (2.4) and $\lambda_{n}\left(\tilde{q}_{0}, h_{m}\right)$ be the $n$-th eigenvalue of the boundary-value problem (2.5), (2.6), (2.7). For a fixed index $n$, if

$$
\lambda_{\mathrm{n}}\left(\mathrm{q}_{0}, \mathrm{~h}_{\mathrm{m}}\right)=\lambda_{\mathrm{n}}\left(\tilde{\mathrm{q}}_{0}, \mathrm{~h}_{\mathrm{m}}\right), \quad \mathrm{m}=1,2, \cdots,
$$

then

$$
\mathrm{q}_{0}(\mathrm{x})=\tilde{\mathrm{q}}_{0}(\mathrm{x}), \quad x \in(0, \pi] .
$$

Proof. Multiplying $(2.1)$ by $y(x, \lambda),(2.5)$ by $\tilde{y}(x, \lambda)$ and subtracting and integrating from 0 to $\pi$, we obtain

$$
\left.\left(\tilde{y} y^{\prime}-y \tilde{y}^{\prime}\right)\right|_{0} ^{\pi}+\int_{0}^{\pi}\left(\tilde{q}_{0}-q_{0}\right) y \tilde{y} d x=0 .
$$

Using $y(0, \lambda)=0, y^{\prime}(0, \lambda)=1$ and $\tilde{y}(0, \lambda)=0, \tilde{y}^{\prime}(0, \lambda)=1$, this yields

$$
\left[\left(\tilde{y}(\pi, \lambda) y^{\prime}(\pi, \lambda)-y(\pi, \lambda) \tilde{y}^{\prime}(\pi, \lambda)\right)\right]+\int_{0}^{\pi}\left(\tilde{q}_{0}-q_{0}\right) y \tilde{y} d x=0
$$

We shall formulate as

$$
\begin{gathered}
Q(x)=\tilde{q}_{0}(x)-q_{0}(x), \\
H(\lambda)=\int_{0}^{\pi}\left(\tilde{q}_{0}-q_{0}\right) y \tilde{y} d x .
\end{gathered}
$$

If the properties of $y(x, \lambda)$ and $\tilde{y}(x, \lambda)$ are considered, the function $H(\lambda)$ is an entire function of order $\frac{1}{2}$ in $\lambda$. We see that

$$
\left[\left(\tilde{y}(\pi, \lambda) y^{\prime}(\pi, \lambda)-y(\pi, \lambda) \tilde{y}^{\prime}(\pi, \lambda)\right)\right]_{\lambda=\lambda_{\mathfrak{m}}\left(q_{0}, h_{\mathfrak{m}}\right)}=0 .
$$


Using estimate (3.1), (3.2), (3.3) we can write the following equation

$$
\mathrm{H}\left(\lambda_{\mathrm{n}}\left(\mathrm{q}_{0}, \mathrm{~h}_{\mathrm{m}}\right)\right)=0 .
$$

From Theorem 2.6, clearly, $\left\{\left(\lambda_{n}\left(q_{0}, h_{m}\right)\right)\right\}_{m=1}^{+\infty}$ is a precisely monotonous and bounded sequence and then there exists a $\lambda_{\mathrm{n}_{0}}\left(\mathrm{q}_{0}\right) \in \mathbb{R}$, satisfies

$$
\lim _{m \rightarrow+\infty} \lambda_{n}\left(q_{0}, h_{m}\right)=\lambda_{n_{0}}\left(q_{0}\right) .
$$

$\lambda_{\mathfrak{n}_{0}}\left(\mathrm{q}_{0}\right)$ is a finite accumulation point of the spectrum set $\left\{\left(\lambda_{\mathrm{n}}\left(\mathrm{q}_{0}, \mathrm{~h}_{\mathrm{m}}\right)\right)\right\}_{\mathrm{m}=1}^{+\infty}$. It is well-known that the set of zeros of every entire function which is not identically zero has not any finite accumulation point. Therefore

$$
H(\lambda)=0, \quad \forall \lambda \in \mathbb{C} .
$$

According to Theorem 2.4 and Theorem 2.5, solutions equations (2.1), (2.5) can be expressed in the following integral forms respectively,

$$
\begin{aligned}
& y(x, \lambda) \equiv \frac{\sin \lambda x}{\lambda}+\int_{0}^{x} K(x, s) \frac{\sin \lambda s}{\lambda} d s+O\left(\frac{e^{|\operatorname{Im} \lambda| x}}{|\lambda|^{6-2 p}}\right), \\
& \tilde{y}(x, \lambda) \equiv \frac{\sin \lambda x}{\lambda}+\int_{0}^{x} \tilde{K}(x, s) \frac{\sin \lambda s}{\lambda} d s+O\left(\frac{e^{|\operatorname{Im} \lambda| x}}{|\lambda|^{6-2 p}}\right) .
\end{aligned}
$$

By means of integral equations (3.5) and (3.6) we get

$$
y \tilde{y}=\frac{\sin ^{2} \lambda x}{\lambda^{2}}+\int_{0}^{x}(\tilde{K}(x, s)+K(x, s)) \frac{\sin \lambda x}{\lambda} \frac{\sin \lambda s}{\lambda} d s+\int_{0}^{x} K(x, s) \frac{\sin \lambda s}{\lambda} d s \times \int_{0}^{x} \tilde{K}(x, t) \frac{\sin \lambda t}{\lambda} d t+O\left(\frac{e^{|\operatorname{Im} \lambda| x}}{|\lambda|^{6-2 p}}\right) .
$$

Using the trigonometric addition formulas, extending the range of $K(x, s), \tilde{K}(x, s)$ with respect to the second argument, we obtain that

$$
y \tilde{y}=\frac{1}{2 \lambda^{2}}\left[1-\cos 2 \lambda x+\int_{0}^{x} \tilde{\mathrm{K}}(x, r) \cos 2 \lambda r d r+O\left(\frac{e^{|\operatorname{Im} \lambda| x}}{|\lambda|^{4-2 p}}\right)\right]
$$

where

$$
\widetilde{\mathrm{K}}(x, s)=2\left[\mathrm{~K}(x, x-2 r)+\tilde{\mathrm{K}}(x, x-2 r)+\int_{-x+2 r}^{x} \mathrm{~K}(x, t) \tilde{\mathrm{K}}(x, t-2 r) d t+\int_{-x}^{x-2 r} K(x, t) \tilde{K}(x, t+2 r) d t\right] .
$$

Substituting (3.7) into (3.4) and (3.3) and letting $\lambda \rightarrow \infty$ for all $\lambda$, by means of Riemann-Lebesgue lemma and applying some straight forward computations, we can find that

$$
\begin{aligned}
& \int_{0}^{\pi} Q\left[1-\cos 2 \lambda x+\int_{0}^{x} \widetilde{\mathrm{K}}(x, r) \cos 2 \lambda r d r\right] d x=0, \\
& \int_{0}^{\pi} Q[1-\cos 2 \lambda x] d x+\int_{0}^{\pi} Q(x) \int_{0}^{x} \widetilde{K}(x, r) \cos 2 \lambda r d r d x=0,
\end{aligned}
$$




$$
\int_{0}^{\pi} \mathrm{Q}(x) \mathrm{d} x=0
$$

and

$$
\int_{0}^{\pi} \cos 2 \lambda x\left[Q(r)-\int_{r}^{\pi} Q(x) \widetilde{\widetilde{K}}(x, r) d x\right] d r=0 .
$$

Taking into account the completeness of the function $\cos 2 \lambda x$, we can write that

$$
\mathrm{Q}(\mathrm{r})-\int_{\mathrm{r}}^{\pi} \mathrm{Q}(\mathrm{x}) \tilde{\widetilde{K}}(x, r) \mathrm{d} x=0, \quad 0<x \leqslant \pi .
$$

Since (3.8) is Volterra integral equation, it has only trivial solution, $Q(x)=0$. Therefore

$$
\mathrm{Q}(\mathrm{x})=\tilde{\mathrm{q}}_{0}(\mathrm{x})-\mathrm{q}_{0}(\mathrm{x})=0,
$$

almost everywhere on $(0, \pi]$. This completes the proof.

\section{Conclusion}

In this paper, a uniqueness theorem has proved for the solution of an inverse spectral problem having singularity type on the interval $(0, \pi]$. This theorem is discussed from a different view point.

\section{References}

[1] V. A. Ambarzumjan, Über eine frage der eigenwerttheorie, Z. Phys., 53 (1929), 690-695. 1

[2] Y. Aygar, E. Bairamov, Jost solution and the spectral properties of the matrix-valued difference operators, Appl. Math. Comput., 218 (2012), 9676-9681. 1

[3] G. Borg, Eine Umkehrung der Sturm-Liouvilleschen Eigenwertaufgabe, Bestimmung der Differentialgleichung durch die Eigenwerte, (German) Acta Math., 78 (1946), 1-96. 1

[4] N. L. Carothers, Real analysis, Cambridge University Press, Cambridge, (2000). 2.1

[5] Z. M. Gasimov, On the determination of the Singular Sturm Liouville differential equation, Academic Congress of Kravchcuk Kiev, (1992). 1, 2.2

[6] Z. M. Gasimov, Solved inverse problems for Singular Sturm Liouville differential equation from two spectra, Ph.D. thesis, Baku State University, (1992). 2.5

[7] Z. M. Gasımov, Inverse problem with two spectra for a singular Sturm-Liouville equation, Dokl. RAN., 365 (1999), 304-305. 1, 2.2

[8] I. M. Gel'fand, B. M. Levitan, On the determination of a differential equation by its spectral function, (Russian) Doklady Akad. Nauk SSSR (N.S.), 77 (1951), 557-560. 1

[9] F. Gesztesy, B. Simon, Inverse spectral analysis with partial information on the potential, II, The case of discrete spectrum, Trans. Amer. Math. Soc., 352 (2000), 2765-2787. 1

[10] O. H. Hald, Inverse eigenvalue problems for the mantle, Geophys. J. Int., 62 (1980), 41-48. 1

[11] H. Hochstadt, B. Lieberman, An inverse Sturm-Liouville problem with mixed given data, SIAM J. Appl. Math., 34 (1978), 676-680. 1

[12] O. R. Hryniv, Y. V. Mykytyuk, Half-inverse spectral problems for Sturm-Liouville operators with singular potentials, Inverse Problems, 20 (2004), 1423-1444. 1, 2

[13] B. M. Levitan, On the determination of the SturmLiouville operator from one and two spectra, Math. USSR Izv., 12 (1978), 179-193. 1

[14] M. M. Malamud, Questions of uniqueness in inverse problems for systems of differential equations on a finite interval, (Russian); translated from Tr. Mosk. Mat. Obs., 60 (1999), 199-258, Trans. Moscow Math. Soc., 1999 (1999), $173-$ 224. 1,2

[15] V. A. Marčenko, Some questions of the theory of one-dimensional linear differential operators of the second order, I, (Russian) Trudy Moskov. Mat. Obšč., 1 (1952), 327-420. 1

[16] J. R. McLaughlin, W. Rundell, A uniqueness theorem for an inverse Sturm-Liouville problem, J. Math. Phys., 28 (1987), 1471-1472. 1 
[17] E. S. Panakhov, E. Baş, On inverse problem for singular Sturm-Liouville operator from two spectra, Proc. Inst. Math. Mech. Natl. Acad. Sci. Azerb., 28 (2008), 85-92. 1, 2.2, 2.4

[18] E. S. Panakhov, M. Sat, Reconstruction of potential function for Sturm-Liouville operator with Coulomb potential, Bound. Value Probl., 2013 (2013), 9 pages. 1

[19] A. N. Tihonov, On the uniqueness of the solution of the problem of electric prospecting, (Russian) Doklady Akad. Nauk SSSR (N.S.), 69 (1949), 797-800. 1

[20] V. Y. Volk, On inversion formulas for a differential equation with a singularity at $x=0$, Uspehi Matem. Nauk (N.S.), 8 (1953), 141-151. 1, 2

[21] Y.-P. Wang, A uniqueness theorem for indefinite Sturm-Liouville operators, Appl. Math. J. Chinese Univ. Ser. B, 27 (2012), 345-352. 1, 2 